\title{
How Does Pubertal \\ Development Impact \\ Caregiver-Adolescent \\ Communication About \\ Sex in Rural, African \\ American Families? \\ An Examination of \\ Mediation Effects
}

\author{
Tiarney D. Ritchwood', Isha W. Metzger', \\ Terrinieka W. Powell ${ }^{2}$, Giselle Corbie-Smith ${ }^{3}$, \\ Mysha Wynn 4 , Feng-Chang Lin ${ }^{3}$, \\ and Aletha Y. Akers ${ }^{5}$
}

\begin{abstract}
\footnotetext{
IMedical University of South Carolina, Charleston, SC, USA

2Johns Hopkins Bloomberg School of Public Health, Baltimore, MD, USA

${ }^{3}$ The University of North Carolina at Chapel Hill, NC, USA

${ }^{4}$ Project Momentum Inc., Rocky Mount, NC, USA

${ }^{5}$ Children's Hospital of Philadelphia, PA, USA
}

This study examined the relationship between pubertal development and type of caregiver-adolescent communication about sex (CACS) among 44I African American caregivers participating in an intervention trial in rural North Carolina. We assessed CACS about general sexual health topics and positive aspects of sexuality. Caregivers' attitudes and self-efficacy for CACS, and open communication style were examined as potential mediators. Caregivers engaged in low levels of communication about sex regardless of type. Among caregivers of males, pubertal development was associated with

Corresponding Author:

Tiarney D. Ritchwood, Duke University, 2200 W Main St, Durham, NC 29405, USA.

Email: tiarney.ritchwood@duke.edu 
greater communication about general sexual health, which was mediated by self-efficacy for CACS. Among caregivers of females, pubertal development was associated with less communication about general and positive sexual health topics; however, there were no mediating factors. These findings highlight the predictors of CACS among young men and women after pubertal onset. Age appropriate, practical guidance for initiating CACS may be critical for ensuring caregiver talk about sex.

\section{Keywords}

pubertal development, communication about sex, sex differences, parents, adolescents

African American adolescents are disproportionately impacted by sexually transmitted infections compared with their peers from other ethnic groups (Centers for Disease Control and Prevention [CDC], 2016). When parental caregivers talk with their children about sex, their children are more likely to delay sex initiation and engage in safer sex practices upon sexual debut (e.g., Akers, Holland, \& Bost, 2011; Guilamo-Ramos et al., 2012). Adolescent engagement in safer sexual practices is most likely to occur when caregiveradolescent communication about sex (CACS) includes discussion about a wide range of sexual health topics, and it is open and honest (e.g., Martino, Elliott, Corona, Kanouse, \& Schuster, 2008; Blinded; Blinded). However, some caregivers never discuss sex or do so infrequently, incompletely, or only after their adolesents begin having sex (e.g., Beckett et al., 2010; Ritchwood et al., 2017, 2018). One study of African American caregivers found that when such discussions did occur, caregivers were selective about the type of information that they shared with adolescents, such that discussions about menstruation, pregnancy, and condom and contraception use were more common than discussions about more sensitive sexual topics, such as sexual desire and pleasure, types of coital acts (e.g., oral, anal, and vaginal sex), masturbation, and nocturnal emissions (Ritchwood et al., 2018). Lack of balanced CACS could leave adolescents unprepared to negotiate safe sexual practices with their sexual partners, leading to heightened sexual risk (Ritchwood et al., 2018; Widman, ChoukasBradley, Helms, Golin, \& Prinstein, 2014). Although a number of studies have explored the factors that influence whether CACS occurs, few have sought to identify what determines the type of information shared.

\section{Pubertal Development and CACS}

One factor that may significantly influence whether caregivers talk with adolescents about sex is their adolescent's pubertal development. The 
physical changes that accompany puberty carry much social significance, as they represent the transition from childhood to adolescence. For caregivers, these physical cues (e.g., breast development for girls, voice deepening for boys) may spark conversations about sexuality. Previous research in Sweden, for example, suggested that caregivers tend to infer social maturity from pubertal development (e.g., Tilton-Weaver, Vitunski, \& Galambos, 2001). Relatedly, caregivers may assume that pubertal development will either accompany or closely follow interest in sex or sex initiation, which could trigger caregivers to engage their youths in CACS. Moreover, adolescents are more likely to express their curiosity about sex during puberty, which could also prompt conversations about sex (e.g., Koo, Rose, Bhaskar, \& Walker, 2012; K. S. Miller et al., 2009; Wilson, Dalberth, Koo, \& Gard, 2010). Caregivers' assumptions are supported by research showing that pubertal timing is associated with the onset of adolescent sexual behaviors (Belsky, Steinberg, Houts, \& Halpern-Felsher, 2010; B. C. Miller, Norton, Fan, \& Christopherson, 1998). In fact, studies have associated early pubertal onset with early sex initiation (see review by Baams, Dubas, Overbeek, \& van Aken, 2015). However, the predominant mechanisms through which pubertal development affects the types of sexual health topics caregivers discuss is not well understood.

\section{Influence of Adolescent Biological Sex on CACS}

Caregivers' and adolescents' biological sex also influence CACS. Within African American families, mothers are usually the primary source of information about sex for both boys and girls, though they tend to report more frequent conversations with their daughters than sons (Kapungu et al., 2010; Moore, Berkley-Patton, Bohn, Hawes, \& Bowe-Thompson, 2015; Williams, Pichon, \& Campbell, 2015). In addition to differences in frequency, the content of discussions has been shown to vary by adolescent's biological sex. A retrospective study of undergraduates, for example, suggested that mothers focused their discussions with their daughters on the consequences of engaging in sexual behavior and promoted abstinence and sexual conservatism, when compared with sons, who were more likely to receive messages encouraging them to "have fun" with sex, yet be safe (e.g., Kincaid, Jones, Sterrett, \& McKee, 2012; Kuhle et al., 2015; Widman, Choukas-Bradley, Noar, Nesi, $\&$ Garrett, 2016). Another study found that, during CACS with their adolescent sons, African American mothers tended to focus their discussions on the consequences of pregnancy (i.e., general sexual health), but tended to avoid discussing individual aspects of sexuality such as nocturnal emissions or spontaneous erections (i.e., positive aspects of sexuality; Kapungu et al., 2010; Ritchwood et al., 2018). 
Pubertal onset could also inspire caregivers to discuss different aspects of sexuality with sons compared with daughters. Namely, caregivers of boys may use pubertal development as a sign that their sons' interest in romantic relationships has or may soon begin and, as a result, caregivers' discussions may reflect an effort to encourage safe and "fun" sexual exploration. Conversely, caregivers of girls tend to avoid discussing the positive aspects of sex, and instead may use pubertal onset as a signal to talk with girls about the consequences of sex. To date, there is little understanding of whether puberty impacts CACS and if so, how pathways differ by adolescents' biological sex. Thus, knowing which topics are discussed with adolescents of each biological sex and how puberty may affect the breadth of topics covered could elucidate these associations.

\section{Theoretical Framework}

The integrated behavioral model (IBM; Fishbein, 2000) provides a useful framework for identifying and understanding factors that influence CACS. According to this model, knowledge, attitudes, normative beliefs, and selfefficacy influence both one's intentions to perform a behavior and the actual performance of the target behavior. When applied to CACS, previous research has linked a number of IBM constructs to caregivers' intentions to talk with their children about sex and actual engagement in CACS, including: knowledge about sexual health, positive attitudes toward CACS, higher self-efficacy to engage in CACS, open communication style, and expectations about the outcome of such conversations (Guilamo-Ramos, Jaccard, Dittus, \& Collins, 2008; Jerman \& Constantine, 2010; Ritchwood et al., 2017; Williams et al., 2015). Relatedly, the IBM framework can also be used to better characterize the association between pubertal development and CACS. For example, a child's level of pubertal development could impact their caregiver's attitudes toward CACS and their self-efficacy with regard to engaging the child in conversations about sex and sexual health. Specifically, caregivers might associate their children's advanced level of pubertal development with readiness for CACS thereby sparking their intentions to engage their children in CACS and influencing their attitudes and self-efficacy for CACS. This could determine whether they actually engage their children in CACS. To date, this pathway has yet to be examined.

\section{Current Study}

The purpose of the current study is to determine whether domains from the IBM that have most consistently been shown to predict CACS mediate the 
relationship between pubertal development and CACS among caregivers of African American youths. We focus on African American youths residing in the rural South because they often experience pubertal development earlier than their peers from other ethnic backgrounds (see Ellis, 2004 for review) and geographic locations (e.g., Milhausen et al., 2003). We examine (a) whether pubertal development is associated with CACS, and if so, (b) how pubertal development impacts the topics discussed, and (c) whether the predictors of type of CACS differ by adolescent biological sex. We hypothesized that more advanced pubertal development would be associated with more communication about general sexual health (e.g., puberty, birth control, healthy relationships, negative consequences of sex including pregnancy) topics and less communication about positive aspects of sexuality (e.g., pleasure, emotional intimacy) for caregivers of females. For caregivers of males, we expected more advanced pubertal development to be associated with more communication about both general sexual health and the positive aspects of sexuality. Given previous findings regarding factors that influence the occurrence of CACS, we also expected that constructs from the IBM (i.e., attitudes and self-efficacy toward CACS, and open communication style) would better explain the association between pubertal development and CACS (Figure 1).

\section{Method}

\section{Sample and Procedure}

The current study uses baseline data from Teach One, Reach One (TORO), a community-based participatory research (CBPR) risk-reduction intervention that trained African American youths and their caregivers as lay health advisors to disseminate health information within their social networks (CorbieSmith et al., 2011, 2010; Dave et al., 2017; Ritchwood et al., 2015). We recruited participants between December 2008 and May 2012 from five rural counties in eastern North Carolina. Study counties had similar ethnic compositions, poverty rates, and population sizes (State Center for Health Statistics, Office of Healthy Carolinians/Health Education, 2012). Youths were eligible to participate if they (a) were between 10 and 14 years of age, (b) identified as African American, and (c) resided in one of the five study counties. Caregivers were eligible to participate if they were (a) above the age of 18 years and (b) were the biological parent, legal guardian, or parental figure for a participating youth.

We recruited participants from churches, schools, and other local organizations by distributing fliers and brochures, and by radio and newspaper 


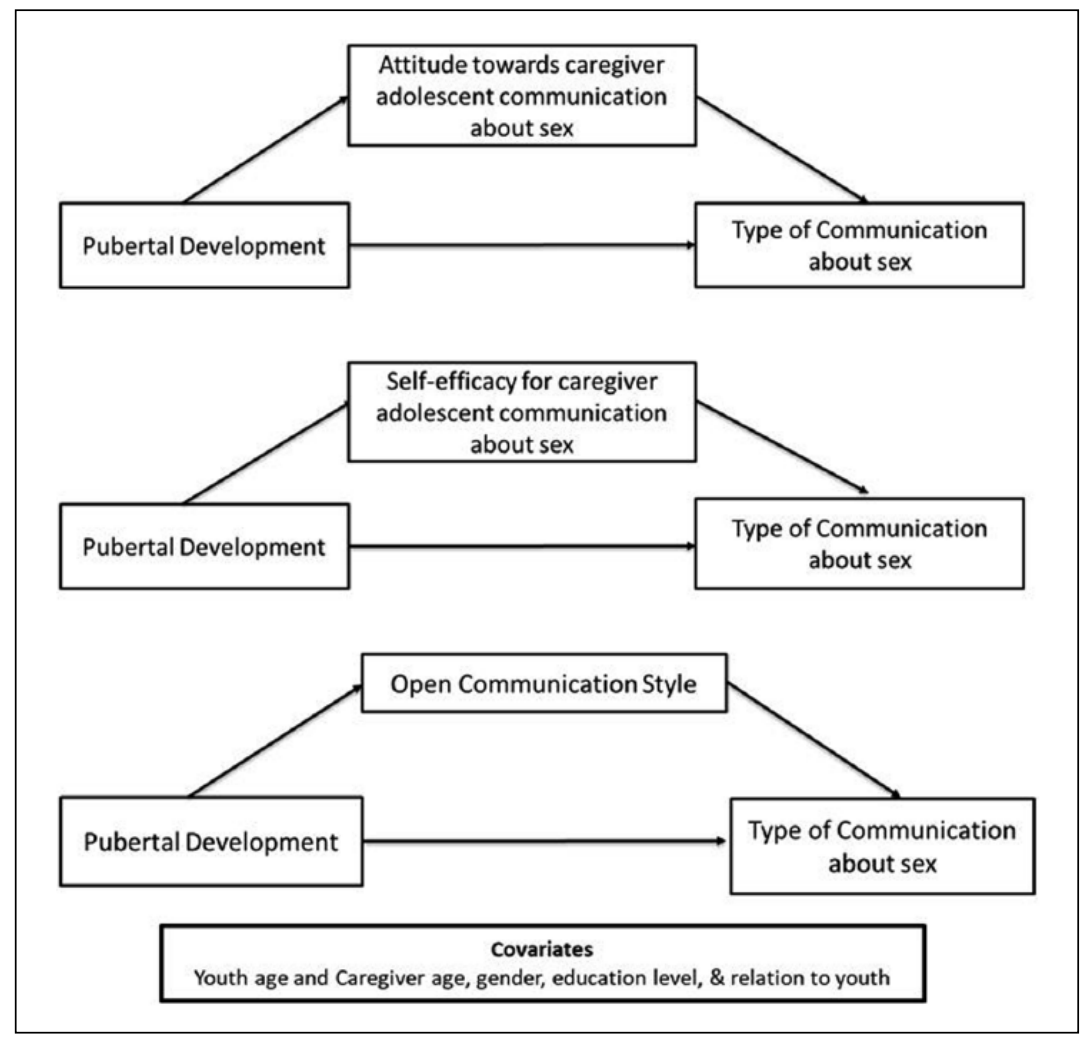

Figure I. Proposed mediators explaining how pubertal development impacts type of caregiver-adolescent communication about general sexual health.

announcements. Potential participants were directed to contact the study office for additional information about inclusion criteria, study goals, and activities. Study staff used a screener to determine participant eligibility, which in addition to the criteria outlined above, included an assessment of characteristics that the community and academic partners agreed were important for lay health advisors (Corbie-Smith et al., 2011, 2010; Ritchwood et al., 2016). Interested and eligible caregivers provided consent for themselves and permission for their youths, while youths provided assent. Both caregivers and youths completed hour-long, self-administered baseline surveys prior to the start of the intervention. To address potential issues regarding confidentiality and low literacy, we used audio computer-assisted self-interview (ACASI). Initially, participants were offered US\$10 cash incentives for participation. 
However, due to difficulty with recruitment, at the recommendation of our community partners, we increased the incentive amount over the baseline period to US $\$ 30$. This study was approved by the institutional review board at a large public university in the southeast region of the United States. Additional details about study recruitment and procedures can be found elsewhere (Corbie-Smith et al., 2011, 2010; Dave et al., 2017; Ritchwood et al., 2015).

\section{Measures}

We used measures validated in African American samples where possible. During the piloting phase of this project, scales were assessed for content validity by means of literature review, and focus groups, surveys, and cognitive interviewing with members of the target population. Individual scale items were summed to create composite scores for each scale. A list of items for scales developed de novo are available from the corresponding author.

Sociodemographics. For the current study, we assessed caregivers' race, age, gender, education, annual income, and relationship to participating youths (e.g., biological parent, legal guardian, other relative). We also assessed youths' age and gender.

CACS. We used the Parent-Adolescent Communication Scale (PACS; Sales et al., 2008), a 20-item scale that assessed caregiver reports of communicating with their youths about sex and sexuality (e.g., "How often you have talked with the youth in the program about ?"). Two items were removed due to nonsignificant correlations with other scale items. Previous research using this scale has indicated that there are two factors: communication about general sexual health $(\alpha=.91)$ and communication about the positive aspects of sexuality $(\alpha=.91)$. The factor that assessed communication about general sexual health consisted of 10 items that assessed discussions about menstruation, physical development, pregnancy, contraception, sex before marriage, and sexuality. The factor that assessed communication about the positive aspects of sexuality consisted of eight items that assessed discussions about sexual satisfaction, types of sex (i.e., vaginal, oral, or anal sex), sexual desire, masturbation, and nocturnal emission. Responses on both subscales range from 0 (never) to 3 (often), with higher scores indicating more frequent communication.

Pubertal development. We used the five-item, Pubertal Development Scale to assess pubertal development for males and females separately $(\alpha=.83$ for males; $\alpha=.69$ for females; Petersen, Crockett, Richards, \& Boxer, 1988). 
All youths were asked whether they had experienced a growth spurt, body hair growth, or androgenic skin changes (e.g., "Have you noticed any skin changes, especially pimples?"). Responses to each of these items were coded on a 4-point scale from 0 (has not yet begun) to 3 (seems completed). The remaining two items were gender-specific and coded either 1 (no) or 4 (yes). Girls were asked whether their breasts had begun to grow, and if they had started to menstruate. Boys were asked whether their voices had begun to deepen, and if they had begun to grow facial hair. Item scores were summed such that a higher score indicated more advanced pubertal development.

Caregiver attitudes toward CACS. Developed de novo, this 6-item scale ( $\alpha=$ .89) assessed caregivers' attitudes about having talking with their early adolescent about sex. Sample items included, "I'd prefer to let the schools teach my child about sex" and "Parents should talk to their child about sexual behaviors." Responses ranged from 0 (strongly disagree) to 3 (strongly agree), and were summed with higher scores indicating more positive attitudes.

Caregiver self-efficacy for CACS. We used the self-efficacy for Parental-Teen Communication Scale (DiIorio et al., 2001) to assess caregivers' confidence in talking with their children about topics of a sexual nature. This 16-item scale $(\alpha=.92)$ included items such as, "You can always explain to the child in the program with you how to use birth control pills." Responses ranged from 0 (not sure at all) to 3 (completely sure), and were summed with higher scores indicating greater self-efficacy.

Caregiver open communication style. We used the 10-item Open Family Communication subscale of the PACS (Barnes \& Olson, 1985), which assessed caregivers' perception of the degree of openness and positive experiences during caregiver-adolescent communication about general topics $(\alpha=.85)$. Sample items included, "I can discuss my beliefs with [my child] without feeling restrained or embarrassed" and, "There are topics I avoid discussing with [my child]." Responses ranged from 0 (strongly disagree) to 3 (strongly agree), and were summed with higher scores indicating more open communication.

\section{Data Analysis}

Data were analyzed using SAS Version 9.4. Descriptive statistics (i.e., frequencies, means, standard deviations) were used to characterize participants. Correlations between the study variables and adolescent biological sex were examined. We used the criteria set by Mackinnon (2008) to determine 
mediation, which indicates that one is able to test for mediation when the independent variable $(X)$ is significantly related to the mediator $(M$; Path $a$ ) and $M$ is significantly related to the dependent variable ( $Y$; Path $b)$. However, a significant direct effect from $X$ to $Y$ (Path $c$ ) is not required. If $X$ remains significant after controlling for $M$, then partial mediation is supported. If $X$ is no longer significant after controlling for $M$, then full mediation is supported (Path $c^{\prime}$ ). We conducted three mediation models for both caregivers of male and female adolescents. Path $a$ was estimated by regressing pubertal development on caregiver attitude toward CACS. We estimated Paths $b$ and $c$, simultaneously, by regressing each of the outcomes (CACS, general sexual health and CACS, positive aspects of sexuality) on pubertal development and caregiver attitude toward CACS. This process was repeated for self-efficacy for CACS and open communication style. For these analyses, we use $p \leqslant .10$ to denote significance. Moreover, bootstrapping methods were used to estimate direct and indirect effects. Bias-corrected accelerated bootstrapping with 5,000 replications was used to obtain $95 \%$ confidence intervals (CIs) around the indirect effects. CIs including zero indicate a nonsignificant effect. In addition, we estimated the indirect mediation effects by using the product of two coefficients in the model pathways (MacKinnon, Lockwood, Hoffman, West, \& Sheets, 2002). Each mediation model was stratified by adolescent biological sex. Models were also adjusted for adolescent age, caregiver age, caregiver biological sex, and caregiver-adolescent relationship status (i.e., biological parent, relative, or other relation).

Handling of missing data. There was little missing data ( $n=441$ complete cases of 465 total cases). Self-efficacy for CACS and communication about the positive aspects of sexuality had the largest amount of missing data $(5 \%$ for each variable). We performed sensitivity testing using multiple imputation and noted the findings were unchanged.

\section{Results}

\section{Descriptives}

The sample characteristics are presented in Table 1. A total of 441 caregivers participated in this study and most were African American, female, approximately 36 years of age, and biologically related to an adolescent participating in the main study. They reported having some college or technical school education, with annual earnings less than US $\$ 40,000$. When compared across adolescent biological sex, the frequency of caregiver reports of caregiveradolescent communication about general sexual health, $t(450)=.38, p=$ 
Table I. Demographic Characteristics of Participants and Mean Scores on Measures $(n=44 I)$.

\begin{tabular}{|c|c|c|}
\hline & Caregivers & Youths \\
\hline Age, $X(S D)$ & $36.2(11.5)$ & $12.5(1.4)$ \\
\hline \multicolumn{3}{|l|}{ Race, \% $(n)^{\mathrm{a}}$} \\
\hline African American & 91.5 (398) & $90.1(392)$ \\
\hline Non-Black & $8.5(37)$ & $9.9(43)$ \\
\hline \multicolumn{3}{|l|}{ Sex, \% (n) } \\
\hline Male & I9.I (83) & $43.7(188)$ \\
\hline Female & $80.9(352)$ & $56.3(242)$ \\
\hline \multicolumn{3}{|l|}{ Relation to adolescent, \% ( $n$ ) } \\
\hline Biological parent & $56.2(242)$ & - \\
\hline Relative & $24.1(104)$ & - \\
\hline Other & $19.7(85)$ & - \\
\hline \multicolumn{3}{|l|}{ Education ${ }^{\mathrm{a}}$} \\
\hline Some high school or less & $22.6(98)$ & - \\
\hline High school diploma & $30.6(133)$ & - \\
\hline Some college/technical school & $27.2(118)$ & - \\
\hline College diploma or higher & $19.6(85)$ & - \\
\hline \multicolumn{3}{|l|}{ Yearly income, \% (n) } \\
\hline$<$ US $\$ 20,000$ & $57.4(227)$ & - \\
\hline US $\$ 20,000-U S \$ 39,999$ & $24.5(97)$ & - \\
\hline US $\$ 40,000-U S \$ 59,999$ & II.I (44) & - \\
\hline US $\$ 60,000-U S \$ 79,999$ & $4.0(16)$ & - \\
\hline US $\$ 80,000$ or more & $3.0(12)$ & - \\
\hline \multicolumn{3}{|l|}{ Measures, $\bar{X}_{\text {(total score) }}(S D$, range) } \\
\hline Caregiver attitudes toward CACS & $15.6(3.0,0-18)$ & - \\
\hline Caregiver self-efficacy for CACS & $39.3(8.4,0-48)$ & - \\
\hline Caregiver open communication style & $21.8(4.4,0-30)$ & - \\
\hline Communication about general sexual health & $17.6(8.5,0-30)$ & - \\
\hline $\begin{array}{l}\text { Communication about positive aspects of } \\
\text { sexuality }\end{array}$ & $6.0(6.3,0-24)$ & - \\
\hline \multirow[t]{3}{*}{ Pubertal development } & $5.0(3.2,0-1 \mathrm{I})$ & - \\
\hline & Male youths & Female youths \\
\hline & $\bar{X}(S D)$ & $\bar{X}(S D)$ \\
\hline Caregiver attitudes toward CACS & $15.7(3.0)$ & $15.3(3.3)$ \\
\hline Caregiver self-efficacy for CACS & $38.7(9.1)$ & $39.6(8.4)$ \\
\hline Caregiver open communication style & $21.9(4.5)$ & $21.7(4.6)$ \\
\hline CAC, general sexual health & $17.6(8.6)$ & $17.4(8.5)$ \\
\hline CAC, the positive aspects of sexuality & $6.1(6.6)$ & $6.2(6.4)$ \\
\hline \multicolumn{3}{|l|}{ Youth report } \\
\hline Pubertal development & $12.7(3.7)$ & $14.9(4.0)$ \\
\hline
\end{tabular}

Note. CACS = caregiver-adolescent communication about sex.

aTotals do not sum to the sample size because of missing data and/or rounding. 
.76 , and the positive aspects of sexuality, $t(438)=.30, p=.70$, did not differ significantly. Attitudes toward CACS, self-efficacy for CACS, and open communication style did not differ between caregivers of males and females ( $p>.05$, respectively). Based on adolescents' reported mean pubertal development score, most adolescents indicated that their pubertal development was "definitely underway." Caregivers reported having a more open communication style, positive attitudes toward CACS, and high self-efficacy for CACS. Despite this, caregivers reported low-to-moderate levels of actual caregiver-adolescent communication about both general sexual health and about the positive aspects of sexuality.

\section{Correlations}

Table 2 contains the results of our correlation analyses by adolescent biological sex. Among caregivers of male adolescents, we found that caregiver age was associated with higher income. Caregiver education was associated with higher income and more positive attitudes toward CACS. More positive attitudes toward CACS were associated with higher caregiver income, greater self-efficacy for CACS, more frequent conversations about general sexual health, and a more open communication style. Self-efficacy for CACS was associated with more frequent conversations about both general sexual health and a more open communication style. More advanced pubertal development was associated with older adolescent age. Greater caregiver communication about general sexual health was associated with greater self-efficacy for CACS, and a more open communication style. Greater caregiver communication about the positive aspects of sexuality was also associated with older adolescent age and a more open communication style. Greater caregiver communication about general sexual health and greater caregiver communication about the positive aspects of sexuality were also correlated.

Among caregivers of females, we found that older caregiver age was associated with older adolescent age, higher income, and more frequent conversations about CACS. Older adolescent age was associated with more advanced pubertal development and more frequent conversations about general sexual health. Higher caregiver education was associated with higher income and a more open communication style. Higher income was also associated with older adolescent age. More positive attitudes toward CACS were associated with higher self-efficacy for CACS, more frequent communication about general sexual health, and a more open communication style. Self-efficacy for CACS was associated with more frequent communication about general sexual health and the positive aspects of sexuality, and a more open communication style. More frequent communication about general sexual health and 


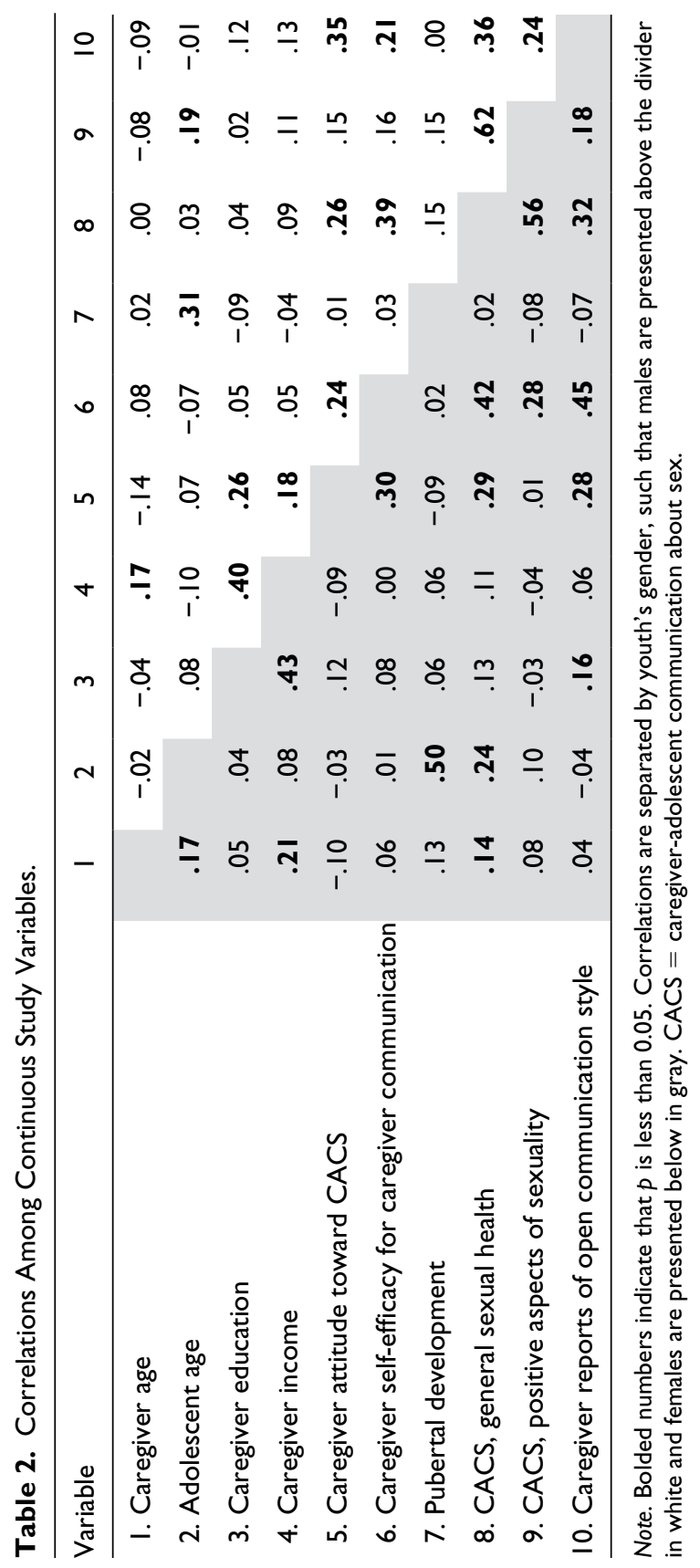


the positive aspects of sexuality was associated with a more open communication style. Greater caregiver communication about general sexual health and greater caregiver communication about the positive aspects of sexuality were moderately correlated.

\section{Mediation Analyses}

Table 3 contains the results of mediation analysis for caregivers of male and female youth.

Caregivers of males. Path $a$ was not significant in any of the tested models. Path $b$, however, was significant for each examined mediator in the CACS about general sexual health models. Though pubertal development was not associated with CACS about general sexual health (Path $c$ ), it was associated with CACS about the positive aspects of sexuality, meaning the more advanced the stage of pubertal development, the more likely it was that communication about the positive aspects of sexuality had occurred. There were no significant indirect effects in examined models.

Caregivers of females. Paths $a$ and $b$ were significant for only one model examining the effect of caregiver attitudes toward CACS on the association between pubertal development and CACS about general sexual health; however, there was no significant indirect effect. Pubertal development was negatively associated with communication about general sexual health, meaning the more advanced the stage of pubertal development the less likely it was that communication about general sexual health had occurred (Path $c$ ). Caregiver attitude toward CACS, self-efficacy for CACS, and open communication style predicted communication about general sexual health (Path $b$ ). Caregiver selfefficacy for CACS and having a more open communication style predicted communication about the positive aspects of sexuality (Path $b$ ).

\section{Discussion}

The primary purpose of the current study was to better understand the relationship between pubertal development and CACS health topics. Our results indicated that, despite caregivers' reports of positive attitudes toward CACS, high self-efficacy for CACS, and an open communication style, few actually talked with the participating adolescents about either general sexual health topics or about the positive aspects of sexuality. For caregivers of boys, more advanced pubertal development was associated with greater communication about the positive aspects of sexuality and there was no association with 


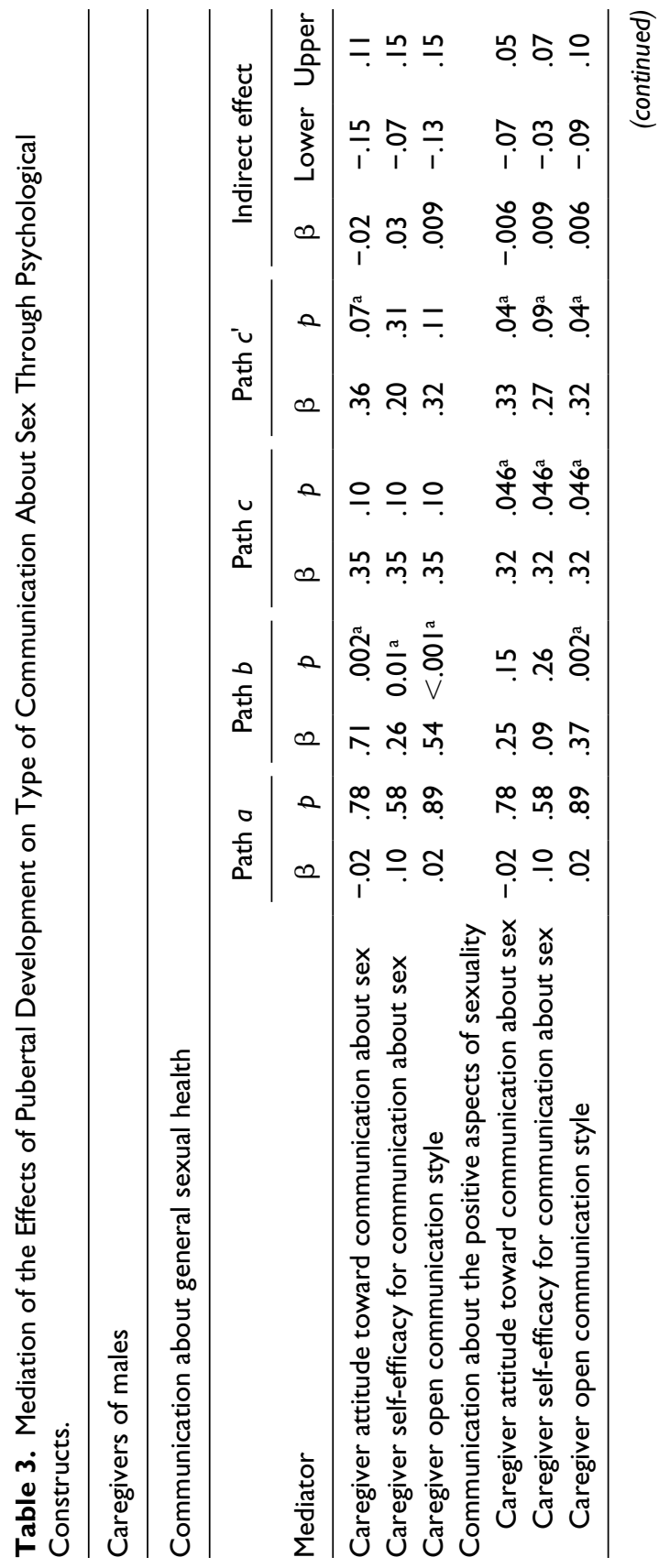




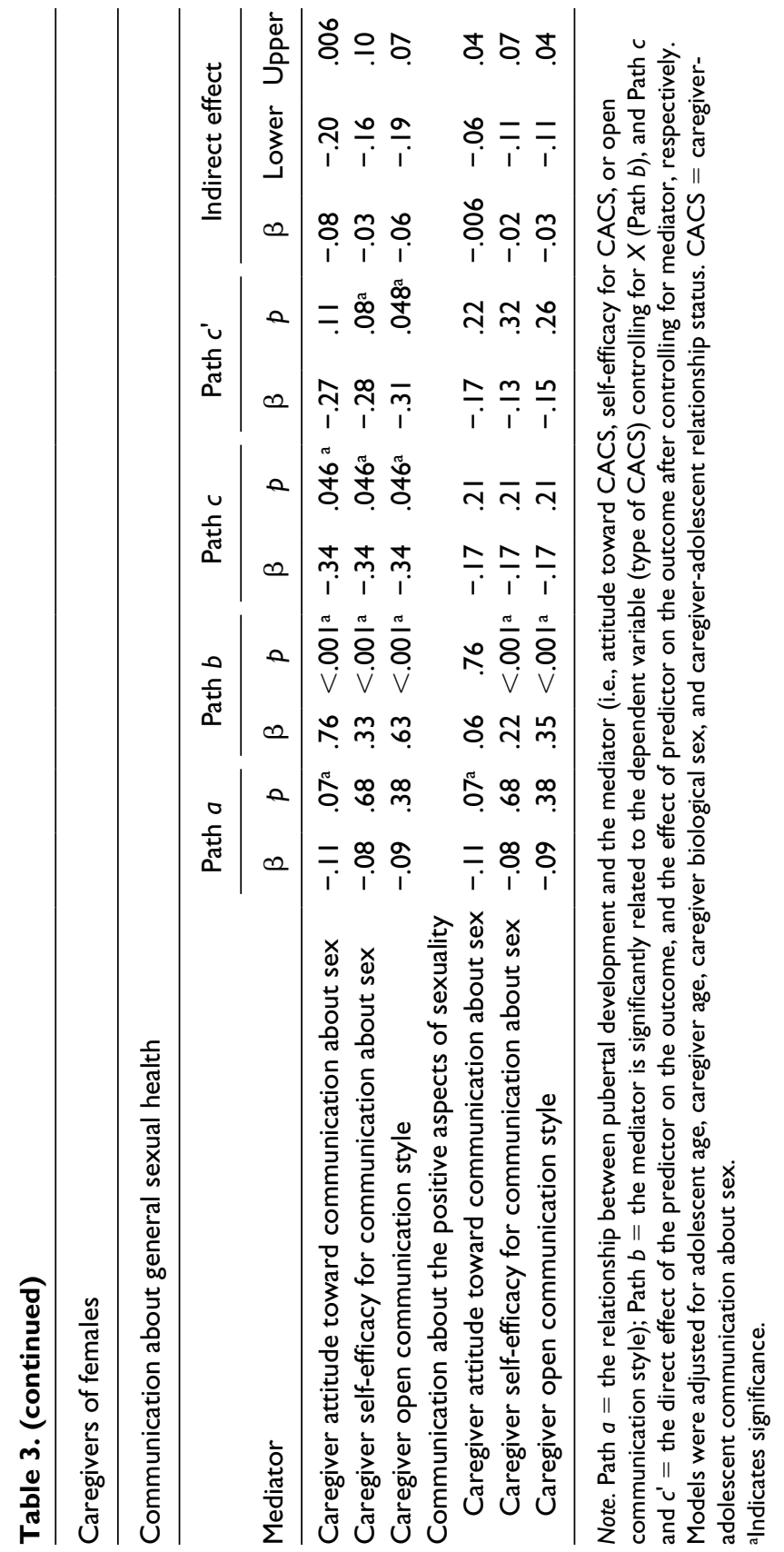


communication about general sexual health topics. For caregivers of girls, pubertal development was associated with less communication about general sexual health topics, and there was no association with communication about the positive aspects of sexuality. There were no significant mediating relationships.

For caregivers of boys, the association between pubertal development and caregiver discussions about the positive aspects of sexuality could suggest that visible signs of pubertal development may be sufficient to cue caregivers that their sons are ready to learn about the positive aspects of sexuality. As pubertal development is often associated with changes in one's social and cognitive maturity, it is possible that caregivers view tangible signs of pubertal development as readiness for CACS. Moreover, caregivers might associate advanced pubertal development with early initiation of sexual behavior and because boys are usually encouraged to practice safe sex rather than to avoid sex altogether, caregivers' discussions of the positive aspects of sexuality may be used to assess their children's knowledge about and interest in sexual behavior, and then to convey information about safe sex. However, such assumptions have not been tested to date. Therefore, more research is needed to further examine this association to better understand and identify mutable factors that influence CACS.

For caregivers of girls, our finding that more advanced pubertal development was associated with less communication about general sexual health conflicts with the conclusions drawn from previous studies suggesting that signs of pubertal development cued mothers to begin talking with their daughters about sex, dating, and relationships (e.g., O'Sullivan, MeyerBahlburg, \& Watkins, 2001). While there are many potential reasons for this finding, it is likely that caregivers' fear of unintentionally encouraging their daughters to engage in early sexual activity serves as a barrier to actual CACS, overriding positive attitudes, higher self-efficacy, and overall good caregiver-adolescent communication (e.g., Wilson \& Koo, 2010). Limited CACS may be a critical issue for caregivers of early African American adolescents in the current study, as most youths reported that their pubertal development was definitely underway. This is an important consideration and a possible area to target in future interventions considering that previous studies have linked pubertal development to sex initiation among early adolescents (Baams et al., 2015). Taken together, our findings suggest the need for interventions to improve caregivers' ability to discuss sexual health topics with adolescent females during the critical period following initiation of pubertal development, when young women's risk of pregnancy begins and their need for information and sexual negotiation skills-building training is greatest. 
We also hypothesized that three domains from the IBM-attitudes and self-efficacy toward CACS as well as having a more open communication style - would mediate the relationship between pubertal development and the types of sexual health topics discussed. We found that none of these consistently mediated the relationship between puberty and CACS for adolescent males and females. Importantly, only caregiver attitudes toward CACS was significantly associated with both pubertal development and CACS, but only when caregivers communicated with girls about general sexual health topics. This finding provides limited support for possibility that caregivers' attitudes could serve as potential barriers to CACS and is consistent with previous research emphasizing the importance of caregivers' confidence in their ability to initiate communication about sex with one's children (e.g., Blinded). These findings extend the existing literature on the influence of attitudes, self-efficacy, and communication style on CACS by demonstrating that these factors may differentially influence communication about various sexual health topics. Moreover, our findings suggest that despite caregivers' positive ratings of their attitudes and self-efficacy for communicating and of their communication style, caregivers may benefit from interventions that increase their actual conversations across a wide range of sexual health topics.

The majority of caregivers in this study reported having an open communication style, positive attitudes about CACS, and high self-efficacy for communicating about sex. Each of these factors also predicted CAC about general sexual health for both caregivers of males and females. These findings are consistent with the IBM (Fishbein, 2000; Fishbein \& Yzer, 2003), as previous research has identified communication style, and attitudes and self-efficacy for CACS as key drivers of the CACS (Guilamo-Ramos et al., 2008; Jerman \& Constantine, 2010; Blinded; Williams et al., 2015). Specifically, caregivers who have positive attitudes about CACS, a stronger relationship with their youths as evidenced by willingness to communicate more openly, and have a stronger belief in their ability to engage their youths in CACS will be more likely to intend to engage their youths in CACS and actually follow through.

Regarding the positive aspects of sexuality, open communication style was a significant predictor for caregivers of both boys and girls. The findings regarding open communication style are expected, as it is possible that willingness to discuss topics that may be embarrassing for both parents and youths is less dependent upon one's belief in their ability to do so or upon their positive attitudes toward it, but is rather dependent upon the strength of the caregiver-adolescent relationship and the degree of mutual trust, which could each reflect whether one speaks openly and honestly (e.g., Markham et al., 2010). Our findings could help explain the results of previous studies 
that have emphasized the importance of relationship quality to adolescent outcomes. One study, for example, linked parent-adolescent relationship quality to adolescent engagement in fewer risk behaviors such as hooking up and alcohol use (Johnson, 2013). Another study found that a more rapid increase in parent-adolescent conflict, but not closeness, was associated with sex initiation by the age of 15 years (McElwain \& Bub, 2018). Taken together, these findings could suggest that youths who perceive their caregivers as more willing to provide them with key information and more willing to treat them as peers may be more likely to disclose sensitive information to their parents leading to a greater degree of closeness and trust (Donovan, Thompson, LeFebvre, \& Tollison, 2017).

Self-efficacy for CACS was an additional predictor of CACS about the positive aspects of sexuality for caregivers of girls. This finding is consistent with previous research emphasizing the importance of parental comfort in the sexual communication between parents and their children (Jerman \& Constantine, 2010). Given that the majority of caregivers in this study were mothers, it is possible that shared experiences with daughters may lead to greater rapport and comfort, thus increasing the importance of self-efficacy for CACS to discussing potentially embarrassing or controversial topics given their children's young age. More research, however, is needed to test these possibilities.

The current study has several limitations. First, it is possible that unmeasured additional variables influenced the association between pubertal development and CACS. For example, parental perception of youth's emotional maturity, along with their gender, might influence both the timing and content of CACS. More studies are needed to examine other factors that influence the relationship between pubertal development and the content of CACS within this population. Second, pubertal development was based upon youths' selfassessment alone. Parents' awareness or perception of their child's development may differ from that of their youths (Baams et al., 2015). Future studies should consider including measures of both caregiver and youth perceptions of the adolescents' pubertal development. Third, the majority of participating caregivers were female, which limited our ability to investigate the effect of caregiver gender on the relationship between pubertal development and CACS. African American fathers play a critical role in adolescent engagement in risk behaviors, such that those with greater presence in their adolescents' lives tend to have children who engage in fewer risk behaviors (Boyd et al., 2006; Fosco et al., 2012; Jordan \& Lewis, 2005; Stanik et al., 2013). Moreover, the majority of participants were also single parents, which limits generalizability to families with other structures. However, this was representative of the target population and of families whose youths are at highest risk of adverse reproductive 
outcomes, such as STIs (sexually transmitted infections) and teen pregnancy. This analysis included baseline data from a proportion of participants who were selected as lay health advisors as part of an intervention study. Caregivers and youths in this sample may possess certain characteristics (e.g., leadership potential, trustworthiness, comfort discussing health-related topics, and availability for and commitment to community-building) that may differ from the general population. Last, our analyses are based upon cross-sectional data, which prohibits us from determining causality and whether identified effects are stable across time, thus our conclusions are atemporal (Winer et al., 2016). Future studies utilizing longitudinal data are needed to confirm the current findings.

The current study makes several contributions to the literature on this topic. To our knowledge, this is the first study to examine both direct and indirect effects of pubertal development on type of CACS among caregivers of African American, early adolescents. Second, this study extends previous research that simply examined links between pubertal development and sex initiation (e.g., Baams et al., 2015) to provide critical information regarding the mechanism through which pubertal development influences (or does not influence) the frequency of CACS for caregivers of males and females. Our use of gendered-stratified models extends previous literature that often combined males and females or focused only on either males or females. This is important, as additional research is needed to understand how caregivers introduce sexual communication, what is said, and how gendered messages influence youths' sexual decision making. Further, findings identify a critical gap: caregivers of African American, early adolescents are giving insufficient attention to communication about sex during this highly influential developmental period. Interventions aimed at improving CACS must provide caregivers with age appropriate, practical guidance for initiating CACS on general and the positive aspects of sexuality while also seeking to improve parents' self-efficacy for CACS and actual initiation and commitment to CACS. Moreover, given our findings concerning the importance of open communication between caregivers and adolescents, as well as related research emphasizing caregiveradolescent relationship quality, future interventions might benefit from assessing relationship quality and include caregiver-adolescent modules aimed at improving dyadic or triadic interactions, along with providing training on general communication skills. Such an approach could help both caregivers and adolescents feel more comfortable engaging in conversations about a broad range of sexual topics, thus allowing interventions to be most beneficial for this population that is of particular risk. 


\section{Acknowledgments}

The authors would like to acknowledge the contributions of our university-community partnership, Project Grace.

\section{Declaration of Conflicting Interests}

The author(s) declared no potential conflicts of interest with respect to the research, authorship, and/or publication of this article.

\section{Funding}

The author(s) disclosed receipt of the following financial support for the research, authorship, and/or publication of this article: This work was funded by grants from the National Center on Minority Health and Health Disparities (R24MD001671) and the University of North Carolina Center for AIDS Research (UNC CFAR P30 AI50410). Dr. Ritchwood was supported by training grants from the National Institute on Drug Abuse (R25DA035692) and the National Institute of Mental Health (R25MH087217, R25MH083635). Dr. Metzger was supported by grants from the National Institute on Drug Abuse (R01DA025616) and the National Institute of Mental Health (T32MH18869). Dr. Corbie-Smith was supported by a mid-career award in patientoriented research from National Heart, Lung, and Blood Institute (K24HL105493).

\section{References}

Ajzen, I. (1985). From intentions to actions: A theory of planned behavior. In J. Kuhl (Ed.), Action control: From cognition to behavior (pp. 11-39). Berlin, Germany: Springer.

Akers, A. Y., Holland, C. L., \& Bost, J. (2011). Interventions to improve parental communication about sex: A systematic review. Pediatrics, 127, 494-510.

Baams, L., Dubas, J. S., Overbeek, G., \& van Aken, M. A. (2015). Transitions in body and behavior: A meta-analytic study on the relationship between pubertal development and adolescent sexual behavior. Journal of Adolescent Health, 56, 586-598.

Baron, R. M., \& Kenny, D. A. (1986). The moderator-mediator variable distinction in social psychological research: Conceptual, strategic and statistical considerations. Journal of Personality and Social Psychology, 51, 1173-1182.

Basen-Engquist, K., Mâsse, L. C., Coyle, K., Kirby, D., Parcel, G., Banspach, S., \& Nodora, J. (1998). Sexual risk behavior beliefs and self-efficacy scales. In C. M. Davis, W. L. Yarber, R. Bauserman, G. Schreer, \& S. Davis (Eds.), Handbook of sexuality-related measures (pp. 541-544). Thousand Oaks, CA: Sage.

Beckett, M. K., Elliott, M. N., Martino, S., Kanouse, D. E., Corona, R., Klein, D. J., \& Schuster, M. A. (2010). Timing of parent and child communication about sexuality relative to children's sexual behaviors. Pediatrics, 125, 34-42.

Belsky, J., Steinberg, L., Houts, R. M., \& Halpern-Felsher, B. L. (2010). The development of reproductive strategy in females: Early maternal harshness $\rightarrow$ earlier 
menarche $\rightarrow$ increased sexual risk taking. Developmental Psychology, 46(1), 120 128.

Centers for Disease Control and Prevention. (2011). Diagnoses of HIV infection and AIDS in the United States and dependent areas, 2009. HIV surveillance report. Retrieved from http://www.cdc.gov/hiv/pdf/statistics_2011_hiv_surveillance _report_vol_23.pdf

Centers for Disease Control and Prevention. (2012). Diagnoses of HIV infection and AIDS among adolescents and young adults in the United States and 5 U.S. dependent areas, 2006-2009. HIV Surveillance Supplemental Report, 17(2), 1-46. Retrieved from http://www.cdc.gov/hiv/topics/surveillance/resources/reports/

Centers for Disease Control and Prevention. (2016). HIV among youth. Retrieved from https://www.cdc.gov/hiv/group/age/youth/index.html

Corbie-Smith, G., Adimora, A. A., Youmans, S., Muhammad, M., Blumenthal, C., Ellison, A., . . . Lloyd, S. W. (2011). Project GRACE: A staged approach to development of a community-academic partnership to address HIV in rural African American communities. Health Promotion Practice, 12, 293-302. doi: $10.1177 / 1524839909348766$

Corbie-Smith, G., Akers, A., Blumenthal, C., Council, B., Wynn, M., Muhammad, M., \& Stith, D. (2010). Intervention mapping as a participatory approach to developing an HIV prevention intervention in rural African American communities. AIDS Education and Prevention, 22, 184-202.

Dave, G., Ritchwood, T., Young, T. L., Isler, M. R., Black, A., Akers, A. Y., . . . Corbie-Smith, G. (2017). Evaluating Teach One Reach One-An STI/HIV riskreduction intervention to enhance adult-youth communication about sex and reduce the burden of HIV/STI. American Journal of Health Promotion, 31, $465-$ 475. doi:10.1177/0890117116669402

Dennis, A. C., \& Wood, J. T. (2012). "We're not going to have this conversation, but you get it": Black mother-daughter communication about sexual relations. Women's Studies in Communication, 35, 204-223.

DiIorio, C., Dudley, W. N., Kelly, M., Soet, J. E., Mbwara, J., \& Sharpe Potter, J. (2001). Social cognitive correlates of sexual experience and condom use among 13- through 15-year-old adolescents. Journal of Adolescent Health, 29, 208-216.

Donovan, E. E., Thompson, C. M., LeFebvre, L., \& Tollison, A. C. (2017). Emerging adult confidants' judgments of parental openness: Disclosure quality and postdisclosure relational closeness. Communication Monographs, 84, 179-199.

Ellis, B. J. (2004). Timing of pubertal maturation in girls: An integrated life history approach. Psychological Bulletin, 130, 920-958.

Fletcher, K. D., Ward, L. M., Thomas, K., Foust, M., Levin, D., \& Trinh, S. (2015). Will it help? Identifying socialization discourses that promote sexual risk and sexual health among African American youth. The Journal of Sex Research, 52, 199-212.

Ge, X., \& Natsuaki, M. N. (2009). In search of explanations for early pubertal timing effects on developmental psychopathology. Current Directions in Psychological Science, 18, 327-331. 
Guilamo-Ramos, V., Bouris, A., Lee, J., McCarthy, K., Michael, S. L., Pitt-Barnes, S., \& Dittus, P. (2012). Paternal influences on adolescent sexual risk behaviors: A structured literature review. Pediatrics, 130, e1313-e1325.

Guilamo-Ramos, V., Jaccard, J., Dittus, P., \& Collins, S. (2008). Parent-adolescent communication about sexual intercourse: An analysis of maternal reluctance to communicate. Health Psychology, 27, 760-769.

Hayes, A. F. (2013). Introduction to mediation, moderation, and conditional process analysis: A regression-based approach. New York, NY: Guilford Press.

Jaccard, J., \& Dittus, P. J. (2000). Adolescent perceptions of maternal approval of birth control and sexual risk behavior. American Journal of Public Health, 90, 1426-1430.

James, J., Ellis, B. J., Schlomer, G. L., \& Garber, J. (2012). Sex-specific pathways to early puberty, sexual debut, and sexual risk taking: Tests of an integrated evolutionary-developmental model. Developmental Psychology, 48, 687-702.

Jerman, P., \& Constantine, N. A. (2010). Demographic and psychological predictors of parent-adolescent communication about sex: A representative statewide analysis. Journal of Youth and Adolescence, 39, 1164-1174.

Johnson, M. D. (2013). Parent-child relationship quality directly and indirectly influences hooking up behavior reported in young adulthood through alcohol use in adolescence. Archives of Sexual Behavior, 42, 1463-1472.

Kapungu, C. T., Baptiste, D., Holmbeck, G., McBride, C., Robinson-Brown, M., Sturdivant, A., . . . Paikoff, R. (2010). Beyond the "birds and the bees": Gender differences in sex-related communication among urban African-American adolescents. Family Process, 49, 251-264.

Kincaid, C., Jones, D. J., Sterrett, E., \& McKee, L. (2012). A review of parenting and adolescent sexual behavior: The moderating role of gender. Clinical Psychology Review, 32, 177-188.

Koo, H. P., Rose, A., Bhaskar, B., \& Walker, L. R. (2012). Relationships of pubertal development among early adolescents to sexual and nonsexual risk behaviors and caregivers' parenting behaviors. The Journal of Early Adolescence, 32, 589-614.

Kuhle, B. X., Melzer, D. K., Cooper, C. A., Merkle, A. J., Pepe, N. A., Ribanovic, A., ... Wettstein, T. L. (2015). The "birds and the bees" differ for boys and girls: Sex differences in the nature of sex talks. Evolutionary Behavioral Sciences, 9, 107-115.

Lehr, S. T., Demi, A. S., DiIorio, C., \& Facteau, J. (2005). Predictors of father-son communication about sexuality. Journal of Sex Research, 42, 119-129.

MacKinnon, D. P. (2008). Introduction to statistical mediation analysis. New York, NY: Lawrence Erlbaum.

MacKinnon, D. P., Lockwood, C. M., Hoffman, J. M., West, S. G., \& Sheets, V. (2002). A comparison of methods to test mediation and other intervening variable effects. Psychological Methods, 7(1), 83-104.

Markham, C. M., Lormand, D., Gloppen, K. M., Peskin, M. F., Flores, B., Low, B., $\&$ House, L. D. (2010). Connectedness as a predictor of sexual and reproductive health outcomes for youth. Journal of Adolescent Health, 46(Suppl. 3), S23-S41. 
Martino, S. C., Elliott, M. N., Corona, R., Kanouse, D. E., \& Schuster, M. A. (2008). Beyond the "big talk": The roles of breadth and repetition in parent-adolescent communication about sexual topics. Pediatrics, 121, e612-e618.

McElwain, A. D., \& Bub, K. L. (2018). Changes in parent-child relationship quality across early adolescence: Implications for engagement in sexual behavior. Youth \& Society, 50, 204-228. doi:10.1177/0044118X15626843

Milhausen, R. R., Crosby, R., Yarber, W. L., DiClemente, R. J., Wingood, G. M., \& Ding, K. (2003). Rural and nonrural African American high school students and STD/HIV sexual-risk behaviors. American Journal of Health Behavior, 27, 373-379.

Miller, B. C., Norton, M. C., Fan, X., \& Christopherson, C. R. (1998). Pubertal development, parental communication, and sexual values in relation to adolescent sexual behaviors. The Journal of Early Adolescence, 18, 27-52.

Miller, K. S., Fasula, A. M., Dittus, P., Wiegand, R. E., Wyckoff, S. C., \& McNair, L. (2009). Barriers and facilitators to maternal communication with preadolescents about age-relevant sexual topics. AIDS and Behavior, 13, 365-374.

Moore, E., Berkley-Patton, J., Bohn, A., Hawes, S., \& Bowe-Thompson, C. (2015). Beliefs about sex and parent-child-church sex communication among churchbased African American youth. Journal of Religion \& Health, 54, 1810-1825.

O’Sullivan, L., Meyer-Bahlburg, H., \& Watkins, B. (2001). Mother-daughter communication about sex among urban African American and Latino families. Journal of Adolescent Research, 16, 269-292.

Petersen, A. C., Crockett, L., Richards, M., \& Boxer, A. (1988). A self-report measure of pubertal status: Reliability, validity, and initial norms. Journal of Youth and Adolescence, 17, 117-133.

Pluhar, E. I., DiIorio, C. K., \& McCarty, F. (2008). Correlates of sexuality communication among mothers and 6-12-year-old children. Child: Care, Health and Development, 34, 283-290.

Ritchwood, T. D., Powell, T. W., Metzger, I. W., Dave, G., Corbie-Smith, G., Atujuna, M., ... Akers, A.Y. (2017). Understanding the relationship between religiosity and caregiver-adolescent communication about sex within African-American families. Journal of Child and Family Studies, 26, 2979-2989. doi:10.1007/ s10826-017-0810-9

Ritchwood, T. D., Peasant, C., Powell, T. W., Taggart, T., Corbie-Smith, G., \& Akers, A. Y. (2018). Predictors of caregiver communication about reproductive and sexual health and sensitive sex topics. Journal of Family Issues, 39, 2207-2231. doi:10.1177/0192513X17741920

Sales, J. M., Milhausen, R. R., Wingood, G. M., DiClemente, R. J., Salazar, L. F., \& Crosby, R. (2008). Validation of a Parent-Adolescent Communication Scale for use in STD/HIV prevention interventions. Health Education \& Behavior, 35 , 332-345.

Tilton-Weaver, L. C., Vitunski, E. T., \& Galambos, N. L. (2001). Five images of maturity in adolescence: What does "grown up" mean? Journal of Adolescence, 24, 143-158. 
Widman, L., Choukas-Bradley, S., Helms, S. W., Golin, C. E., \& Prinstein, M. J. (2014). Sexual communication between early adolescents and their dating partners, parents, and best friends. The Journal of Sex Research, 51, 731-741.

Widman, L., Choukas-Bradley, S., Noar, S. M., Nesi, J., \& Garrett, K. (2016). Parentadolescent sexual communication and adolescent safer sex behavior: A metaanalysis. JAMA Pediatrics, 170, 52-61.

Williams, T. T., Pichon, L. C., \& Campbell, B. (2015). Sexual health communication with religious African American families. Health Communication, 30, 328-338.

Wilson, E. K., Dalberth, B. T., Koo, H. P., \& Gard, J. C. (2010). Parents' perspectives on talking to preteenage children about sex. Perspectives on Sexual and Reproductive Health, 42, 56-63.

Wilson, E. K., \& Koo, H. P. (2010). Mothers, fathers, sons, and daughters: Gender differences in factors associated with parent-child communication about sexual topics. Reproductive Health, 7, Article 31.

Winer, E. S., Cervone, D., Bryant, J., McKinney, C., Liu, R. T., \& Nadorff, M. R. (2016). Distinguishing mediational models and analyses in clinical psychology: Atemporal associations do not imply causation. Journal of Clinical Psychology, 72, 947-955.

\section{Author Biographies}

Tiarney D. Ritchwood, $\mathrm{PhD}$, is an assistant professor in the Department of Community and Family Medicine at Duke University. Her research seeks to understand how individual and sociostructural factors interact to influence adolescent progression along the HIV care continuum and develop multilevel interventions to address challenges.

Isha W. Metzger, $\mathrm{PhD}$, is an assistant professor in the Department of Psychology at the University of Georgia. Her research interests center on understanding how risk and resilience factors impact youth outcomes (e.g., HIV contraction, unintended pregnancies, sexual assault, drunk-driving accidents, and legal system involvement).

Terrinieka W. Powell, PhD, is an assistant professor in the Department of Population, Family, and Reproductive Health at Bloomberg School of Public Health at Johns Hopkins University. Her interests focus on using community-based participatory research (CBPR) and qualitative methods to understand how school, home, and faith settings affect the health outcomes of African American youth.

Giselle Corbie-Smith, MD, MSc, is professor of social medicine at the UNC-Chapel Hill School of Medicine. She directs the program on health disparities at the Cecil G. Sheps Center and the Center for Health Equity Research. Her research focuses on engaging minority and underserved communities in research in health disparities and health equity research.

Mysha Wynn, MA, is the founder and executive director of Project Momentum, Inc., a community-based organization. She serves as a community expert for NC Translational and Clinical Sciences Institute (TraCS), the CTSA at UNC-Chapel Hill, 
and has also served as a community mentor for a Kellogg Health Scholar/Postdoctoral Fellow.

Feng-Chang Lin, $\mathrm{PhD}$, is a research assistant professor and biostatistician in the TraCS at the University of North Carolina at Chapel Hill. His primary area of research lies in the development of novel statistical methods for modeling recurrent events that frequently appear in biomedical studies.

Aletha Y. Akers, MD, MPH, is an adolescent gynecologist and medical director of Adolescent Gynecology Consultative Services in the Craig-Dalsimer Division of Adolescent Medicine at The Children's Hospital of Philadelphia. Her research examines the psychosocial determinants of adolescent sexual and reproductive decisions, with an emphasis on family, partner, and peer influences. 